\title{
The Effect of Exercise on Parkinson's Disease
}

\section{Zeinab Rezaee', Sayed Mohammad Marandi ${ }^{*}$, Hojjatallah Alaei², Fahimeh Esfarjani ${ }^{1}$}

${ }^{1}$ Department of Exercise Physiology, Faculty of Physical Education and Sport Sciences, University of Isfahan, Isfahan, Iran

${ }^{2}$ Department of Physiology, School of Medicine, Isfahan University of Medical Sciences, Isfahan, Iran

\section{A BSTRACT}

Introduction: The prevalence of early symptoms of Parkinson's disease, even in middle age and adolescence, has caused widespread concern. Physical activity is known as one of the non-pharmacological methods to reduce behavioral, motor, biochemical, cardiovascular, and mitochondrial dysfunctions in Parkinson's disease. Although the basis of such biological and molecular benefits is unknown, the antioxidant and anti-inflammatory properties of physical activity may improve the symptoms of Parkinson's disease. Conclusion: Although there is no definitive cure for Parkinson's disease, the use of medications along with adjunctive therapies, such as exercise, can be resulted in promising improvements. However, more research is required to achieve an exercise program that provides optimal benefits to patients' quality of life and reduces mortality.

*Corresponding Author: Sayed Mohammad Marandi

E-mail: s.m.marandi@spr.ui.ac.ir 


\title{
تأثير ورزش در بيمارى ياركينسون
}

\author{
زينب رضايى'، سيد محمد مرندى '*، حجت الله علايى '، فهيمه اسفرجانى'

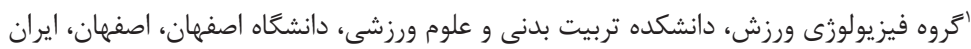

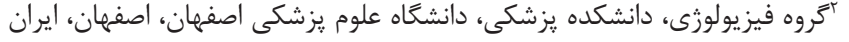

مقدمه: رواج علائم اوليئ بيمارى پاركينسون، حتى در سنين ميانسالى و جوانى، سبب بروز نخرانيهاى

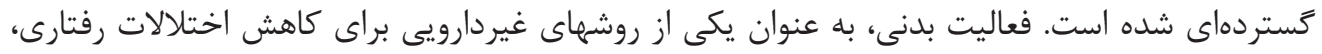

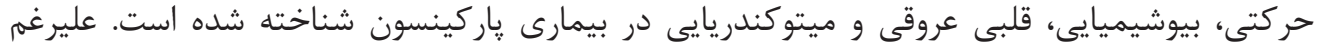

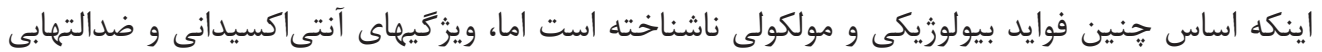

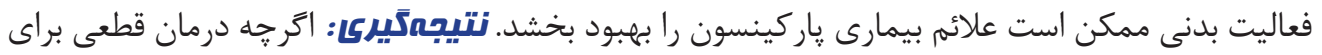

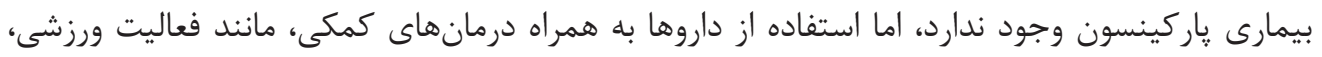

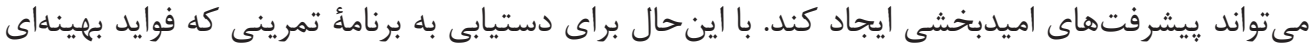

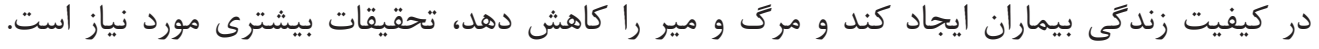

:

ا. ورز

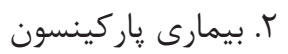

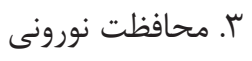

*ونيسنده مسئول: سيد محمد مرندى يست الكترونيك: s.m.marandi@spr.ui.ac.ir 


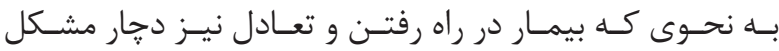

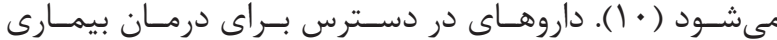

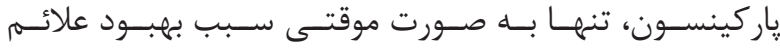

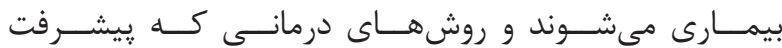

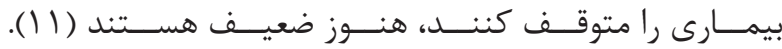

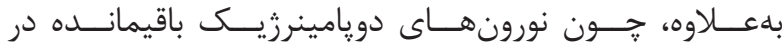

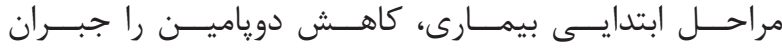

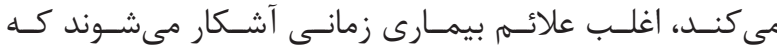

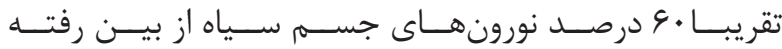

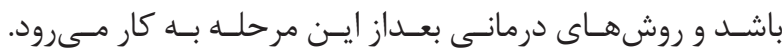

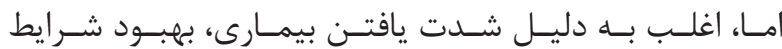

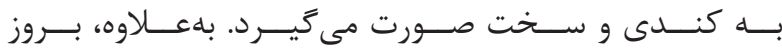

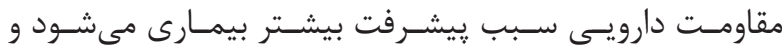

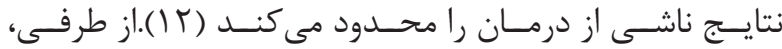

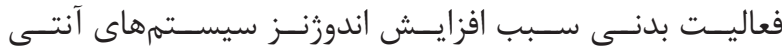

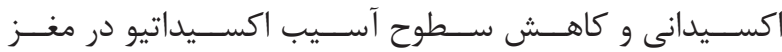

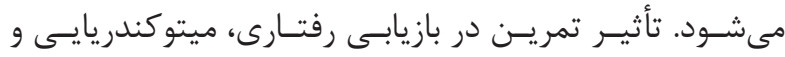

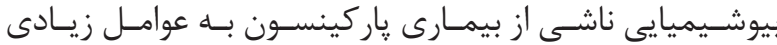

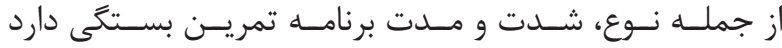

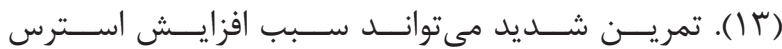

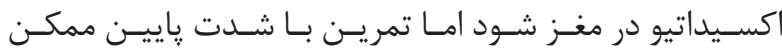

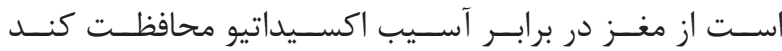

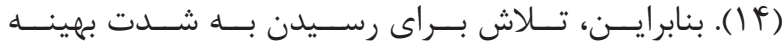

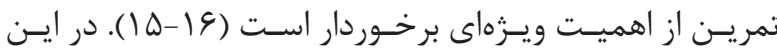

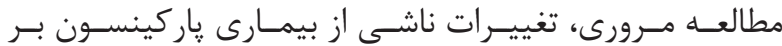

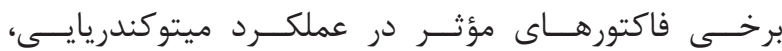

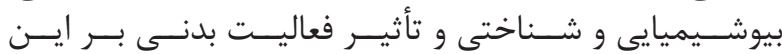

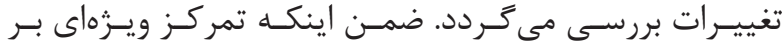

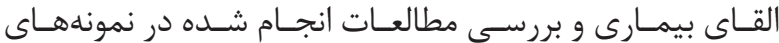

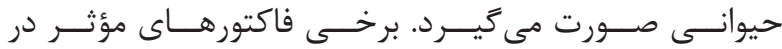

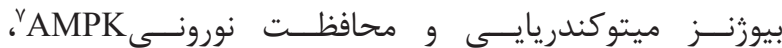

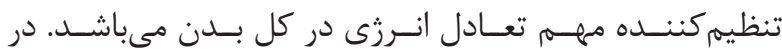

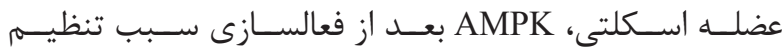

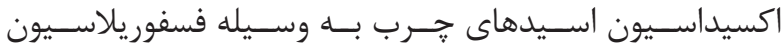

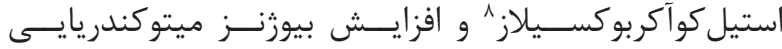

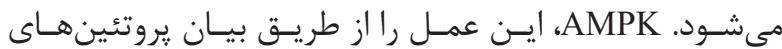

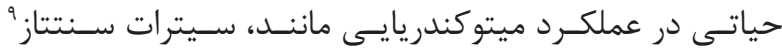

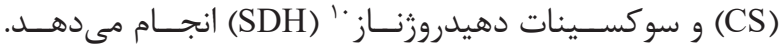

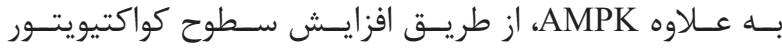

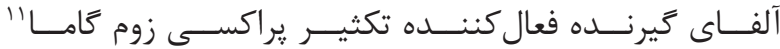

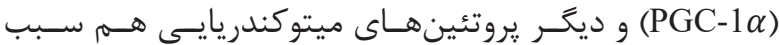

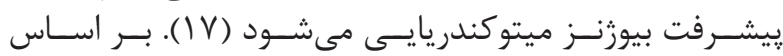

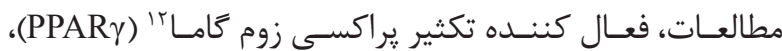

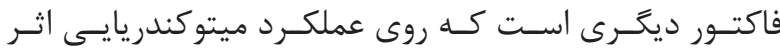

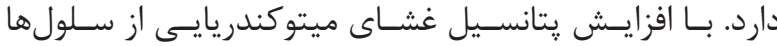

${ }^{1}$ Substantia Nigra Pars Compacta

${ }^{2}$ Inclusion

${ }^{3}$ Lewy Bodies; LBs

${ }^{4}$ Reactive oxygen species; ROS

${ }^{5} \alpha$-Sinuclein

${ }^{6}$ Basal Ganglia
بــا افزايـش ســن، احتمـــال ابتــلا بــهـ اختــلالات شـــاختى در

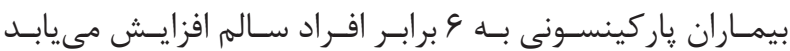

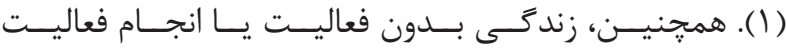

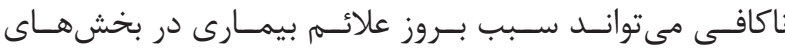

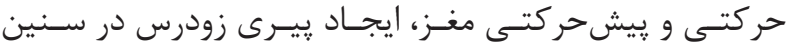

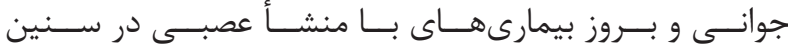

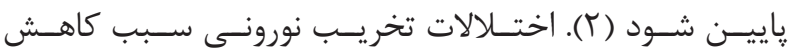

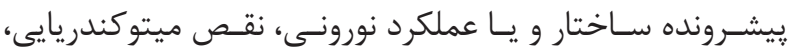

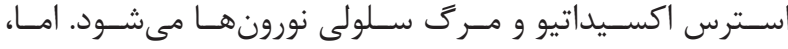

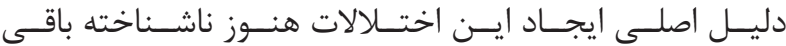

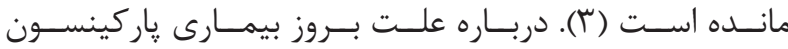

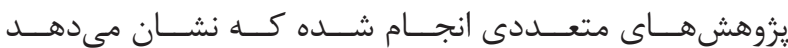

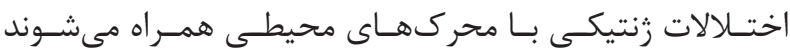

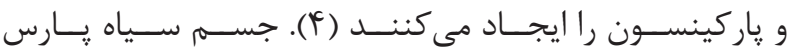

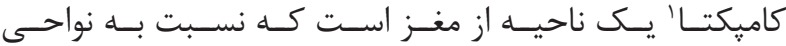

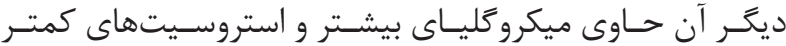

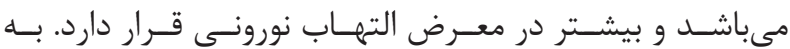

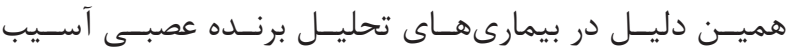

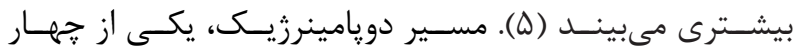

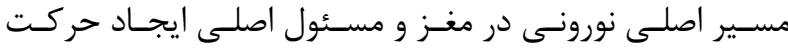

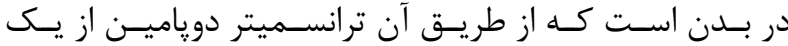

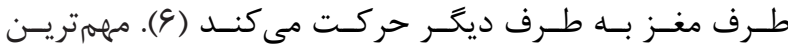

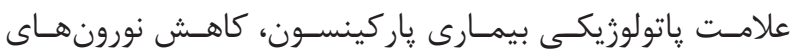

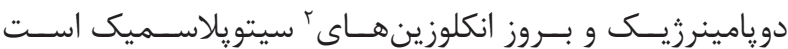

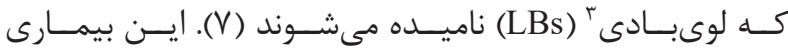

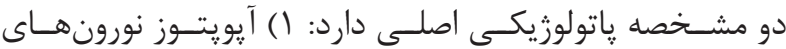

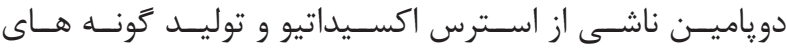

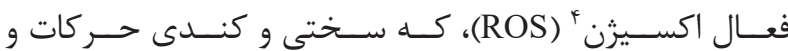

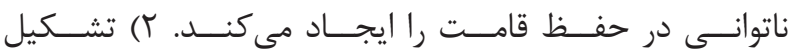

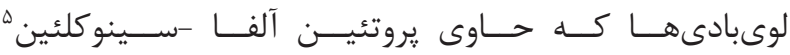

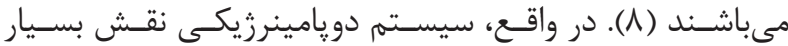

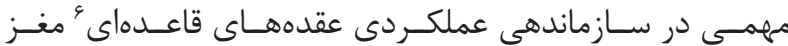

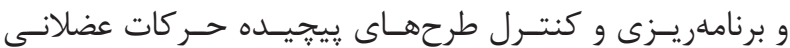

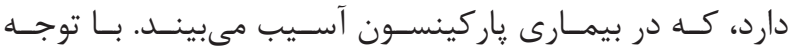

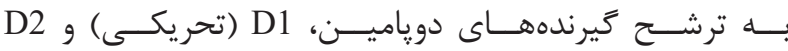

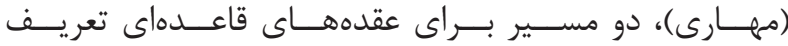

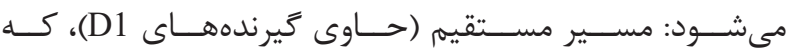

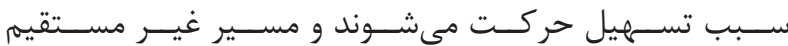

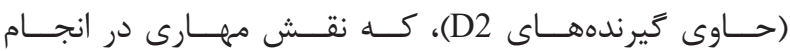

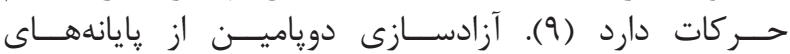

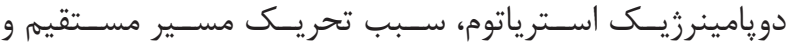

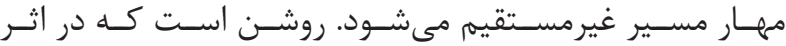

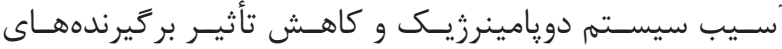

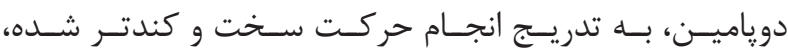
${ }^{7}$ Adenosine Monophosphate-Activated Protein Kinase
${ }^{8}$ Acetyl-CoA carboxylase
${ }^{9}$ Citrate Synthase; CS
${ }^{10}$ Succinate Dehydrogenase; SDH
${ }^{11}$ Peroxisome Proliferator-Activated Receptor Gamma Coactivator 1-Alpha
${ }^{12}$ Peroxisome proliferator-activated receptor gamma 


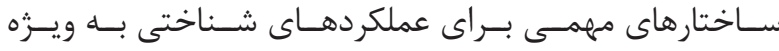

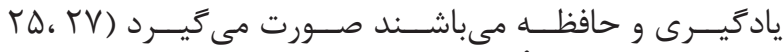

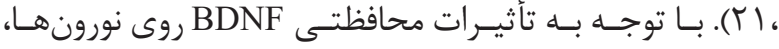

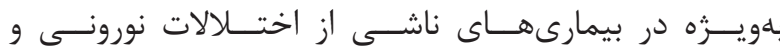

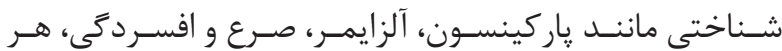

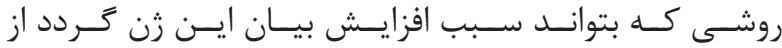

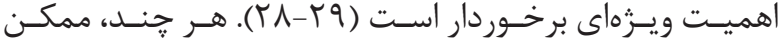

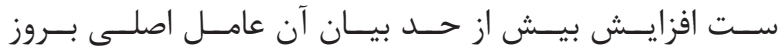

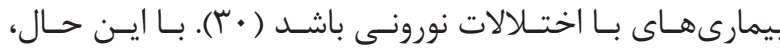

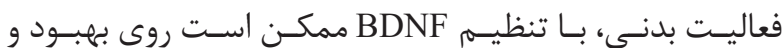

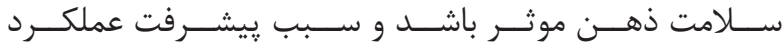

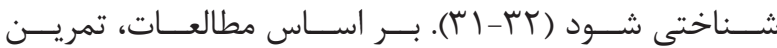

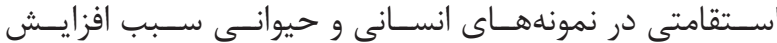

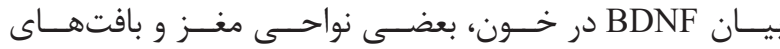

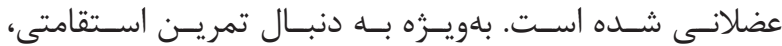

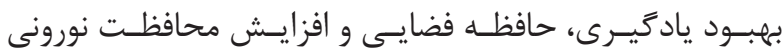

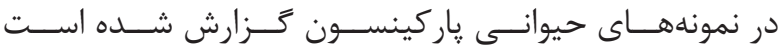

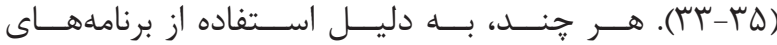

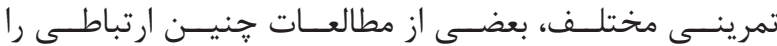

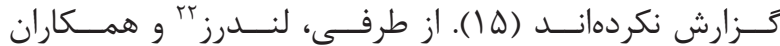

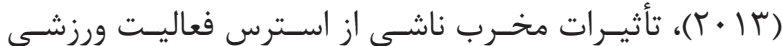

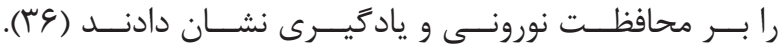

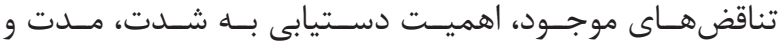

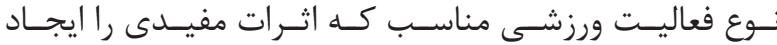

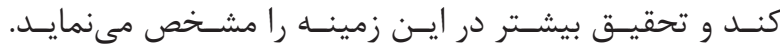

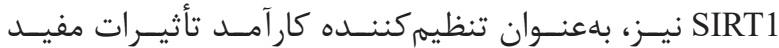

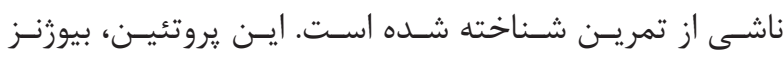

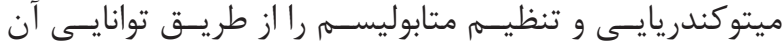

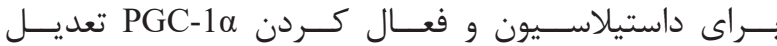

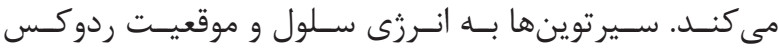

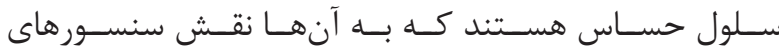

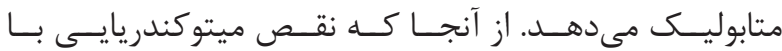

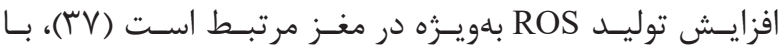

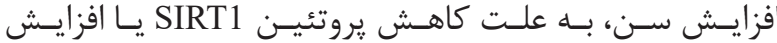

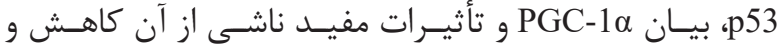

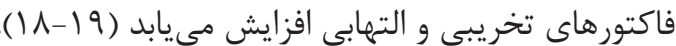

\section{فعاليت بدنى و بيوزنز ميتوكندريايى}

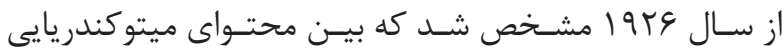

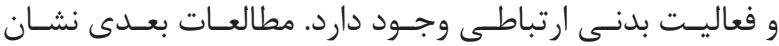

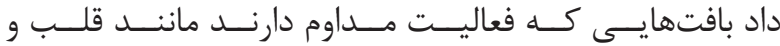

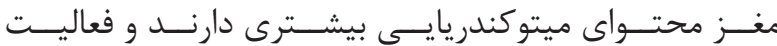

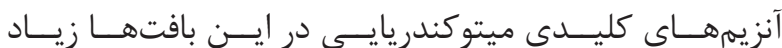

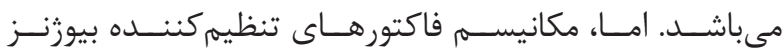

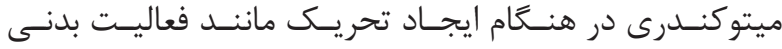

${ }^{13}$ Nuclear Respiratory Factor-1; 2 \& NRF-1

${ }^{14}$ Mitochondrial Transcription Factor A; TFAM

${ }^{15}$ Silent Mating Type Information Regulation 2 Homolog; SIRT1

${ }^{16}$ Reznick

${ }^{17}$ Myokine

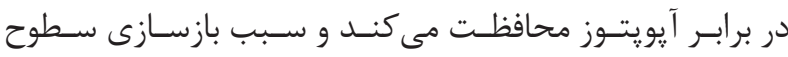

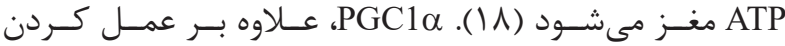

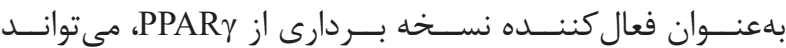

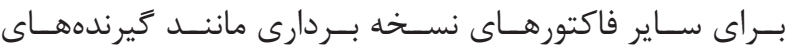

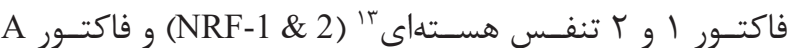

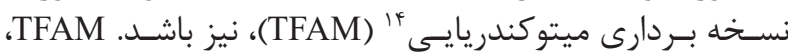

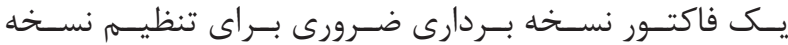

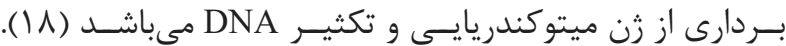

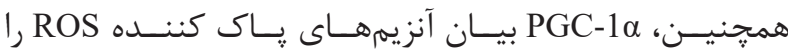

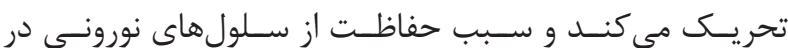

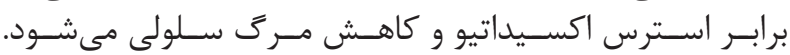

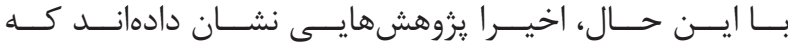

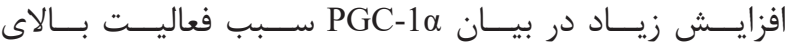

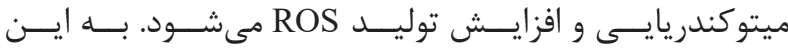

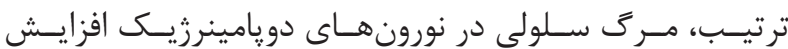

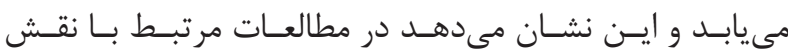

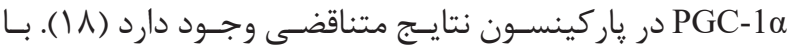

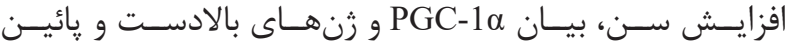

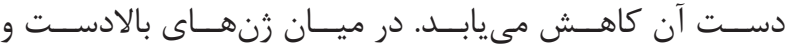

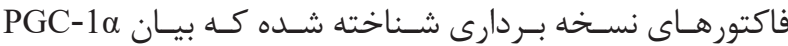

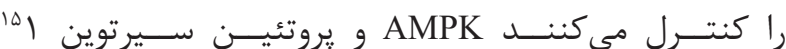

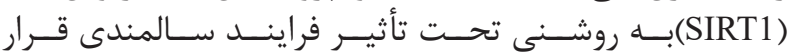

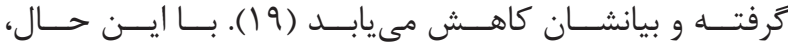

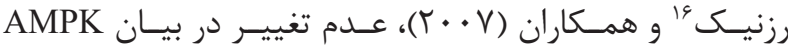

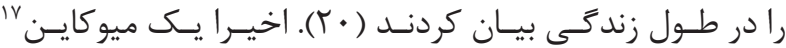

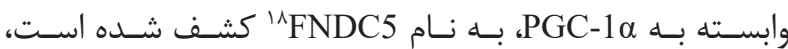

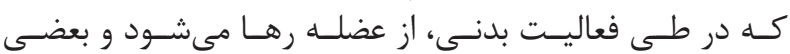

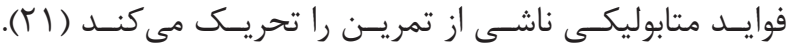

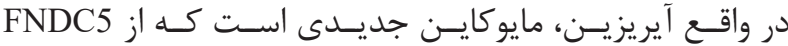

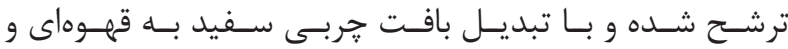

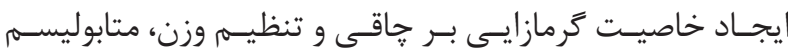

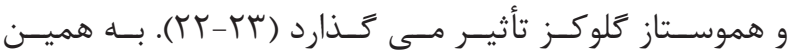

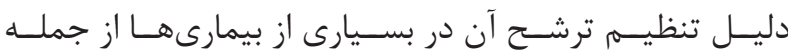

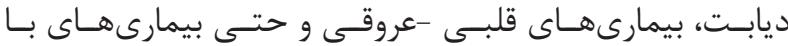

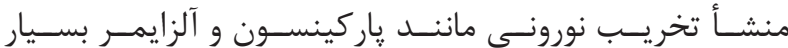

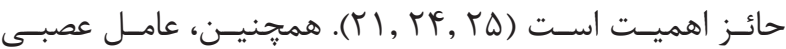

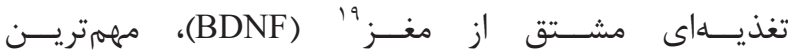

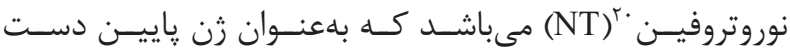

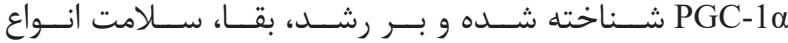

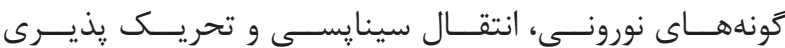

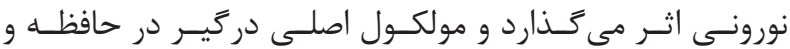

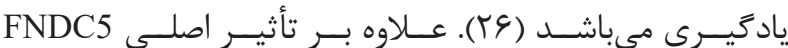

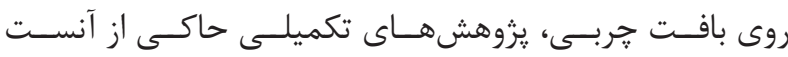

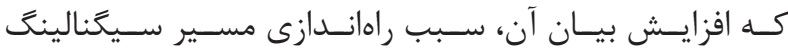
PGC-1 $\alpha /$ FNDC5/BDNF

${ }^{18}$ Fibronectin Type Iii Domain-Containing Protein 5

${ }^{19}$ Brain Derived Neurotrophic Factor; BDNF

${ }^{20}$ Neurotrophin; NT

${ }^{21}$ Hippocampus

${ }^{22}$ Landers 
مسـير سـيخنالينگ PGC-1

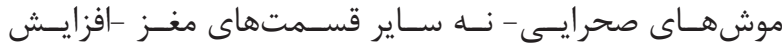

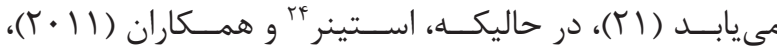

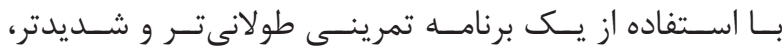

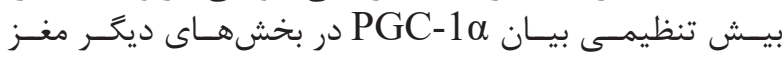

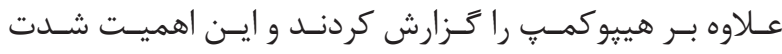

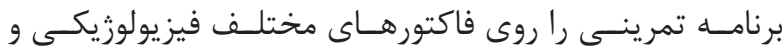

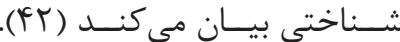

\section{القاى بيمارى پاركينسون در مدلهاى حيوانى}

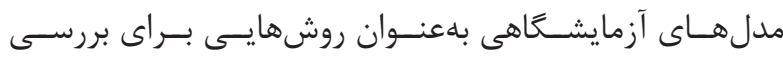

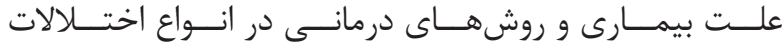

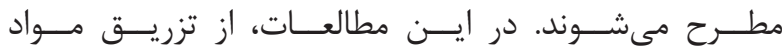

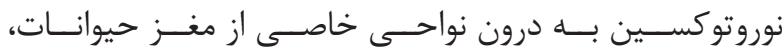

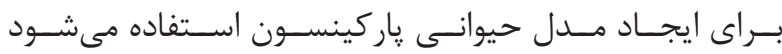

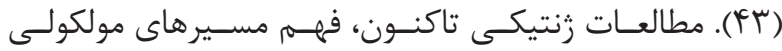

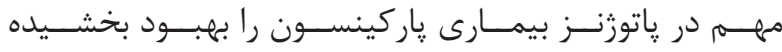

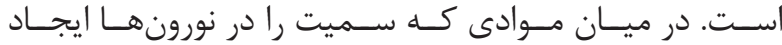
مى كنـنـــ MPTP

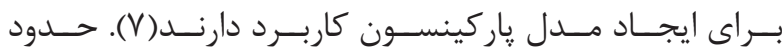

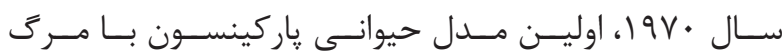

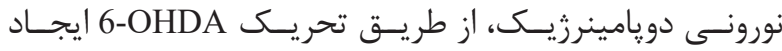

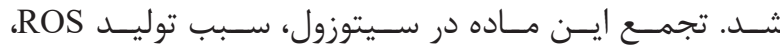

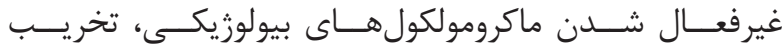

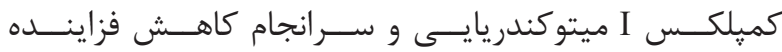

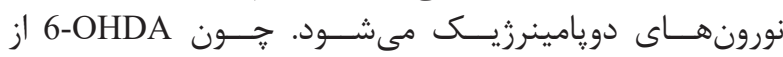

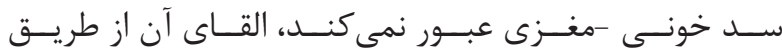

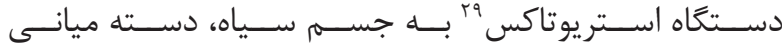

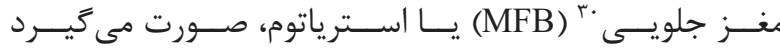

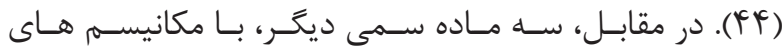

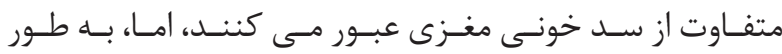

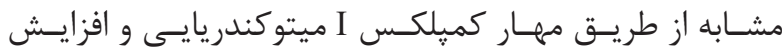

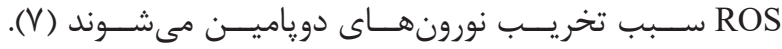

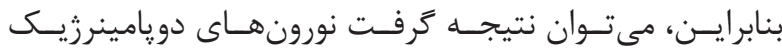

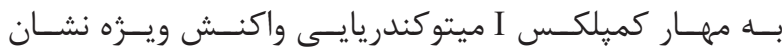

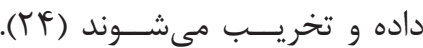

\section{فعاليت بدنى در مدلهاى حيوانى بيمارى پاركينسون}

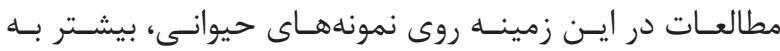

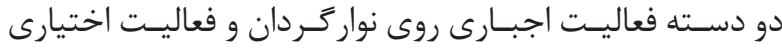

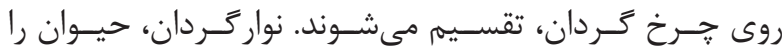

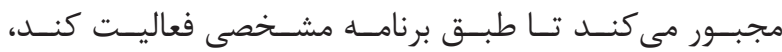

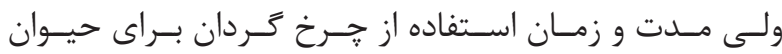

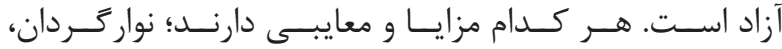

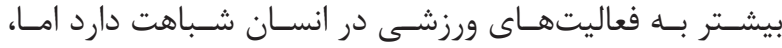

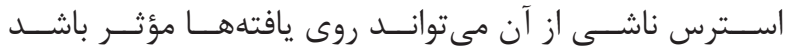

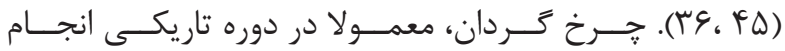

${ }^{23}$ Wrann

${ }^{24}$ Steiner

${ }^{25}$ 6-hydroxydopamine

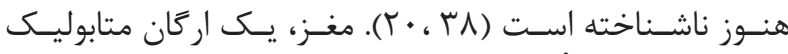

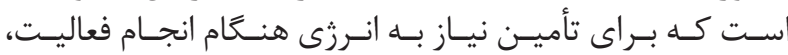

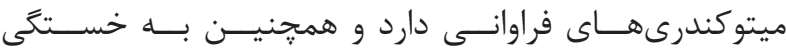

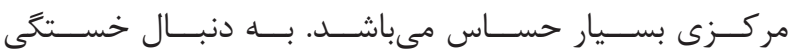

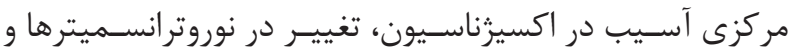

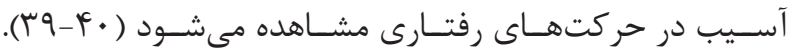

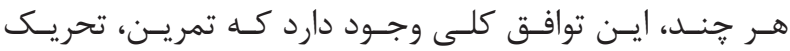

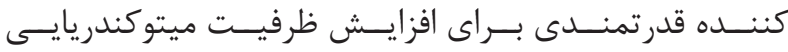

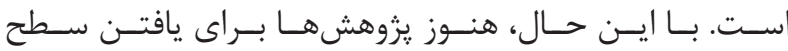

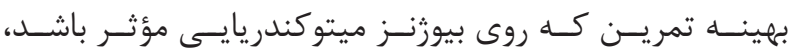

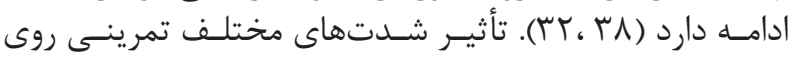

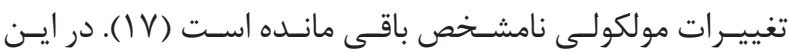

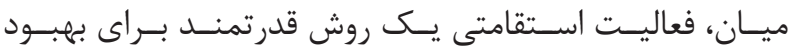

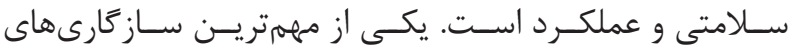

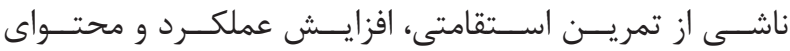

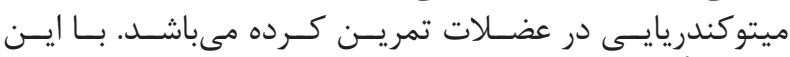

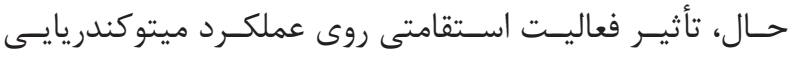

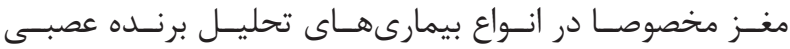

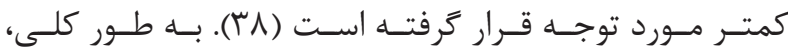

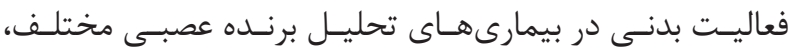

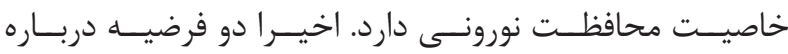

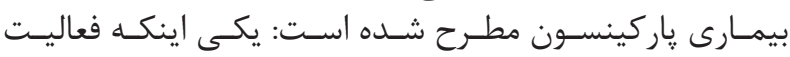

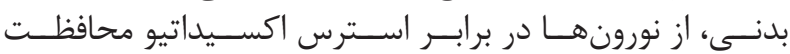

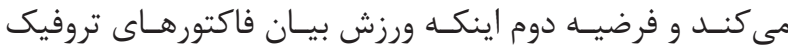

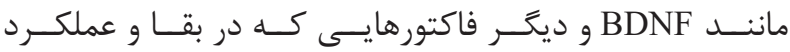

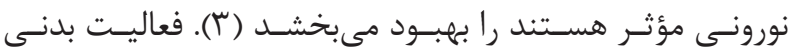

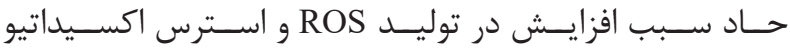

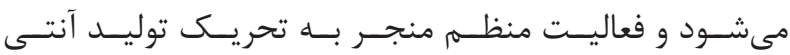

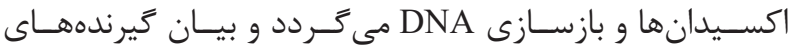

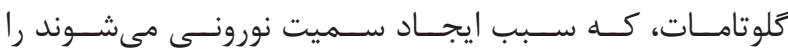

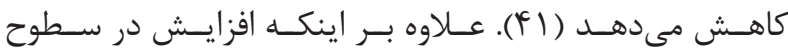
BDNF

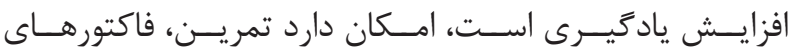

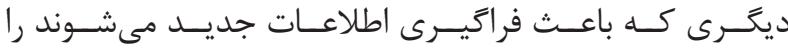

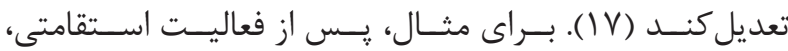

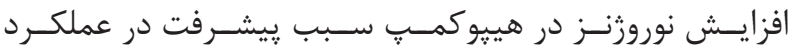

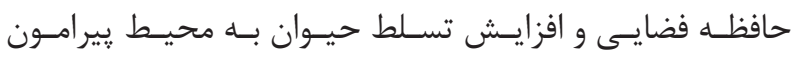

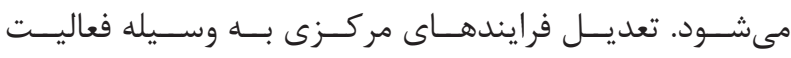

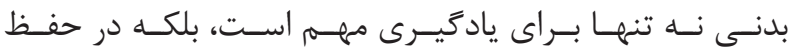

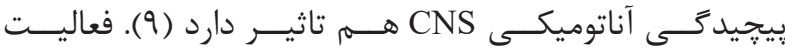

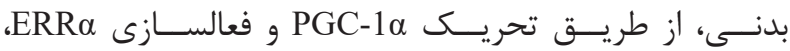

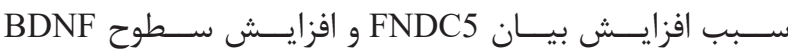

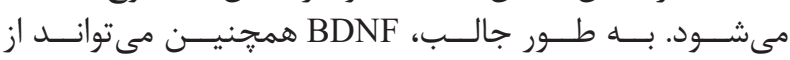

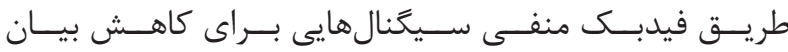
جNDC5

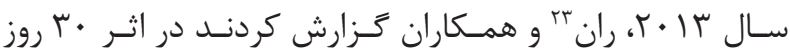

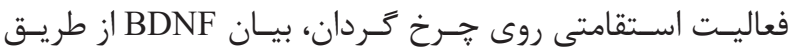

${ }^{26}$ 1-methyl-4-phenyl-1, 2, 3, 6-tetrahydropyridine

${ }^{27}$ Paraquate

${ }^{28}$ Rotenone

${ }^{29}$ Stereotaxy

${ }^{30}$ Medial Forebrain Bundle; MFB 


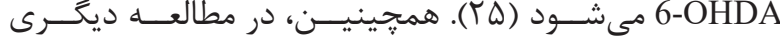

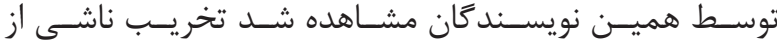

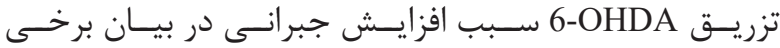

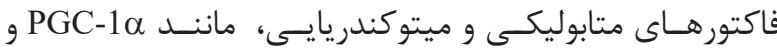
SIRT1

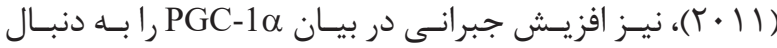

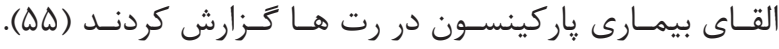

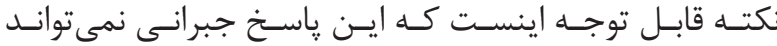

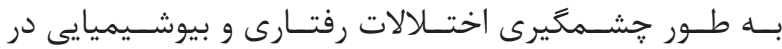

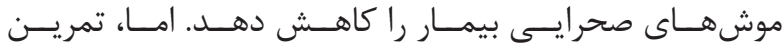

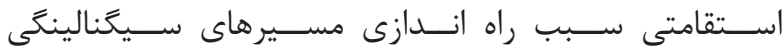

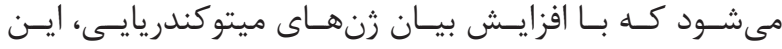

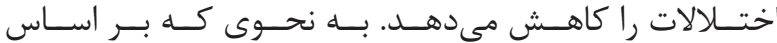

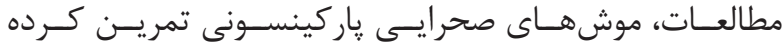

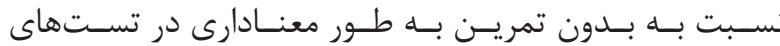

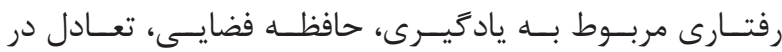

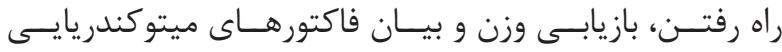

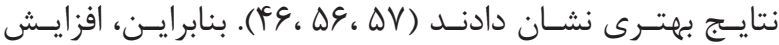

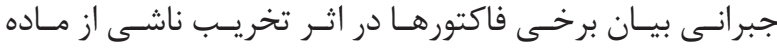

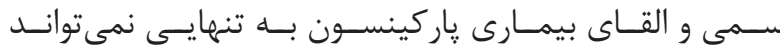

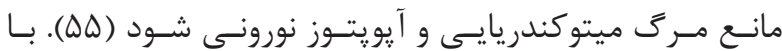

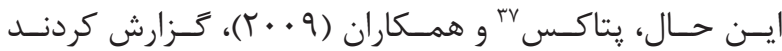

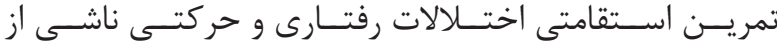

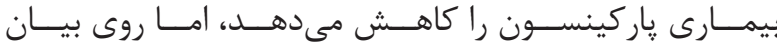

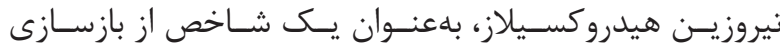

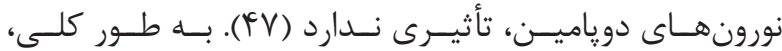

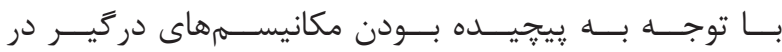

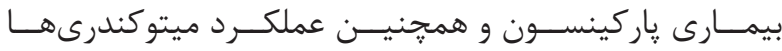

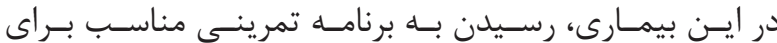

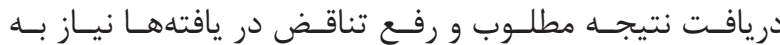

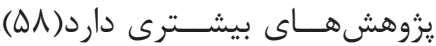

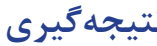

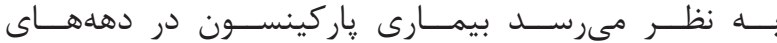

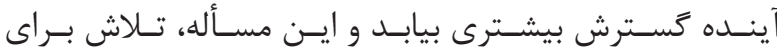

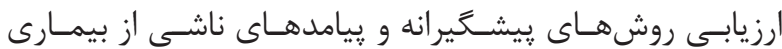

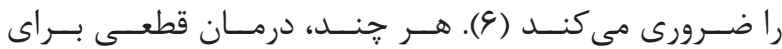

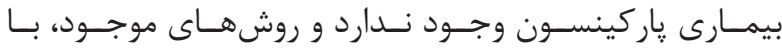

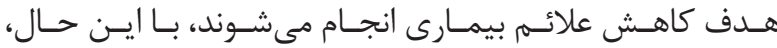

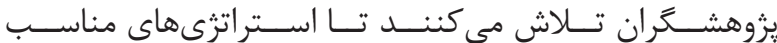

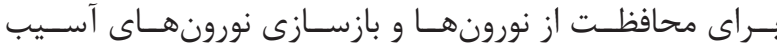

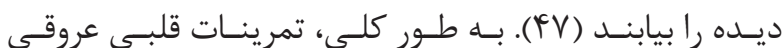

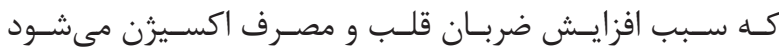

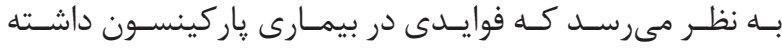

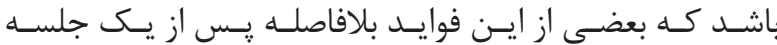

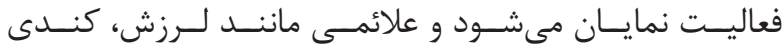

${ }^{31}$ Deane

${ }^{32}$ Poulton and Muir

${ }^{33}$ Gereck

${ }^{34}$ Tyrosine Hydroxyls

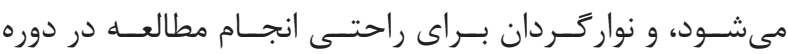

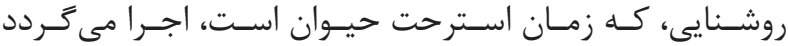

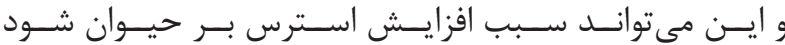

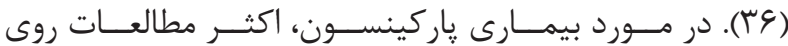

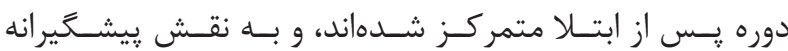

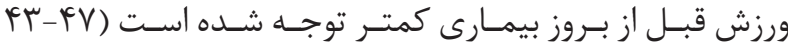

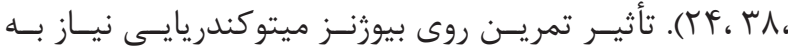

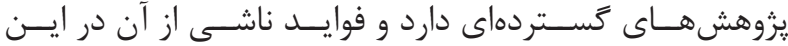

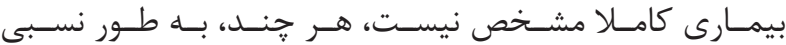

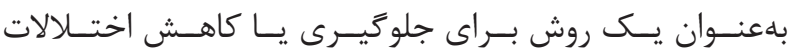

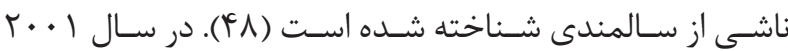

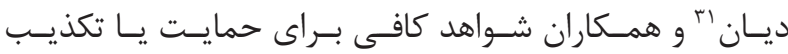

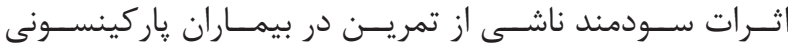

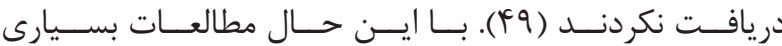

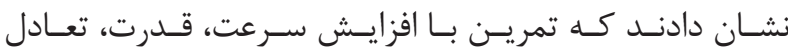

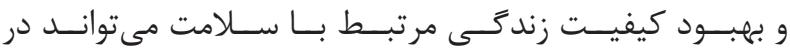

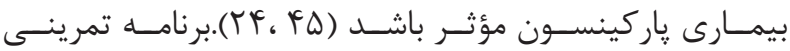

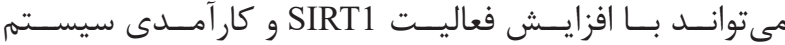

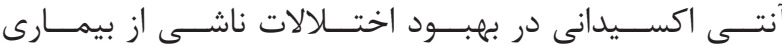

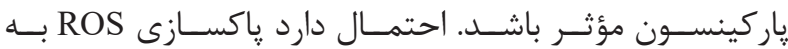

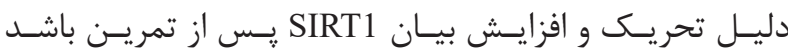

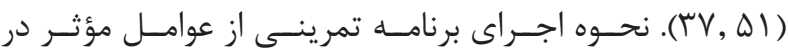

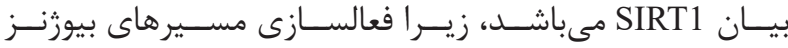

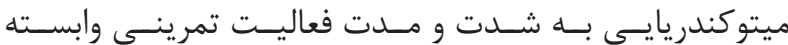

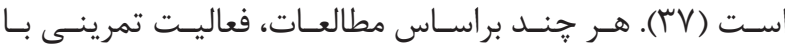

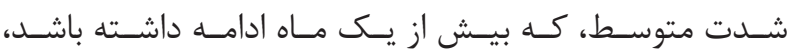

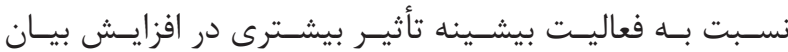

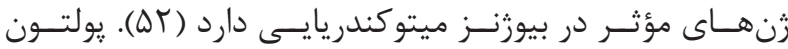

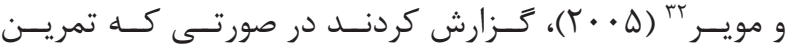

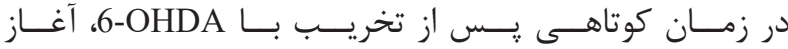

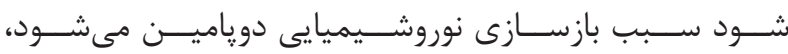

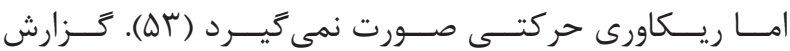

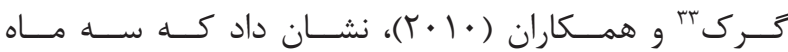

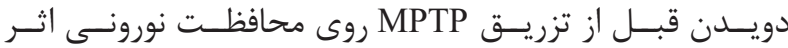

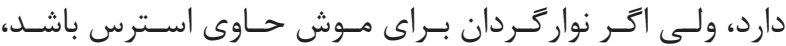

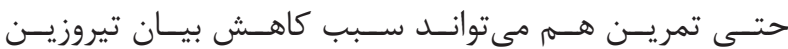

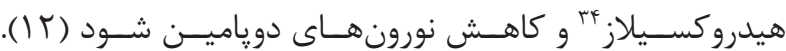

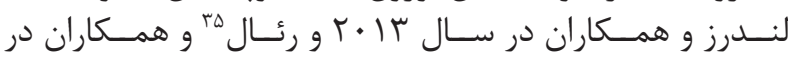

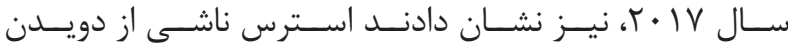

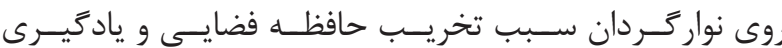

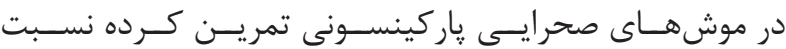

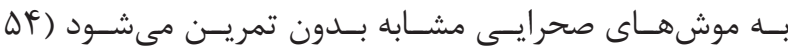

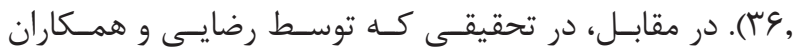

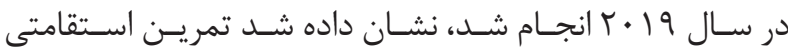

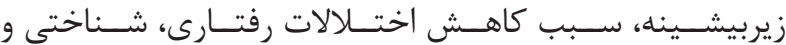

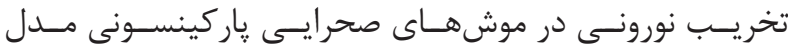

${ }^{35}$ Real

${ }^{36}$ Patki \& Lau

${ }^{37}$ Pothakos 


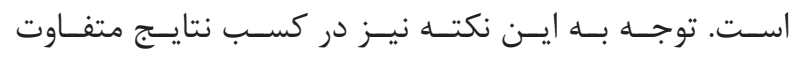

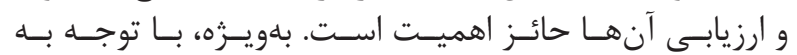

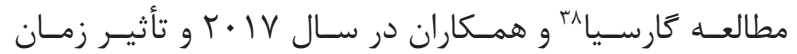

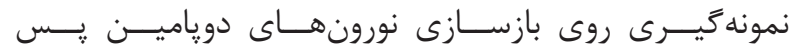

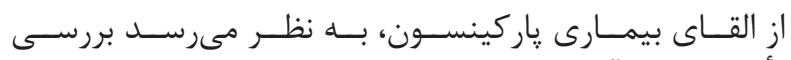

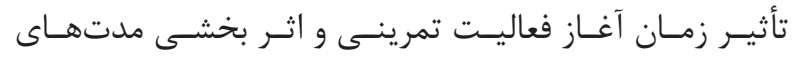

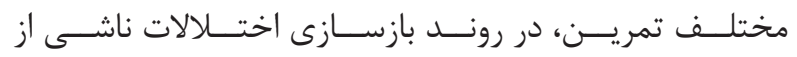

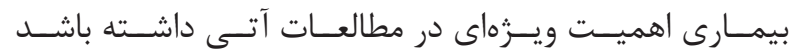

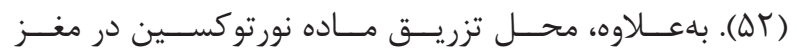

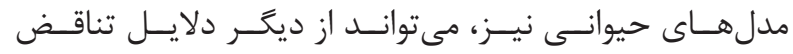

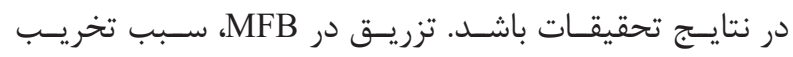

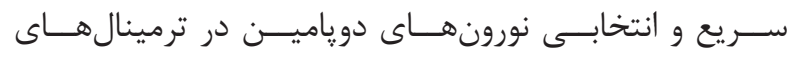

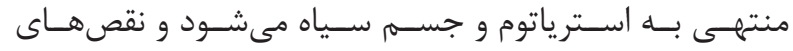

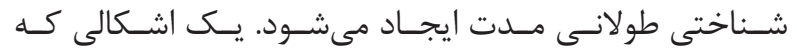

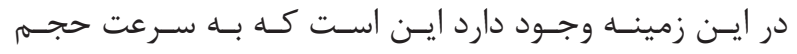

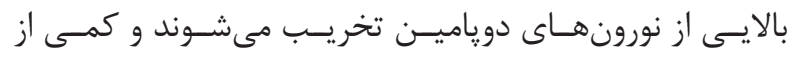

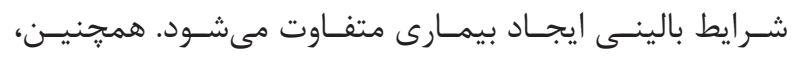

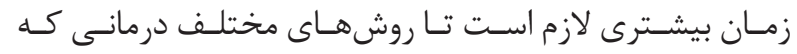

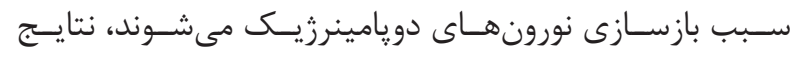

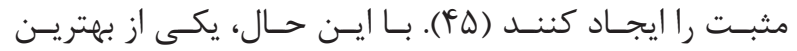

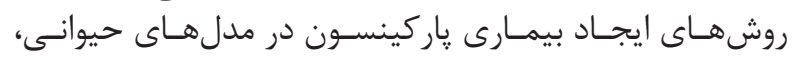

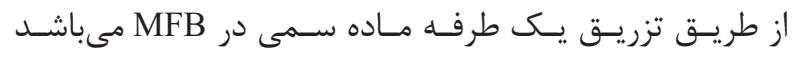

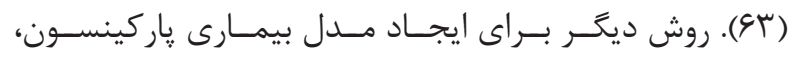

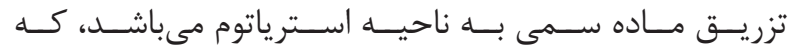

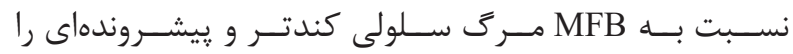

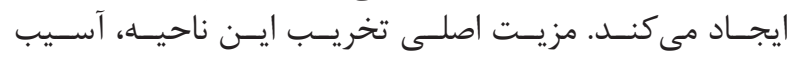

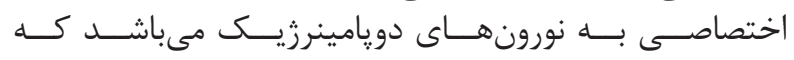

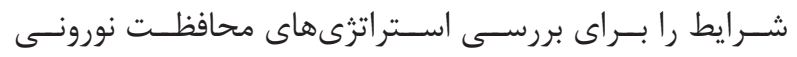

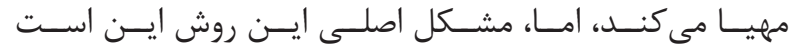

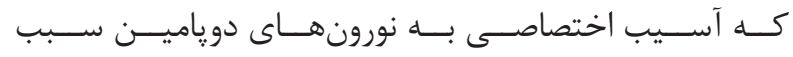

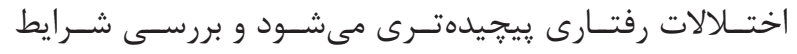

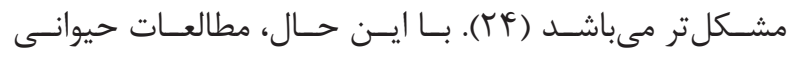

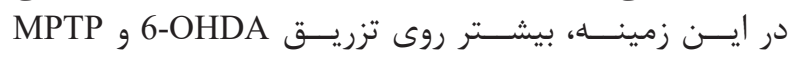

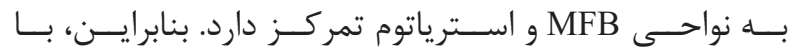

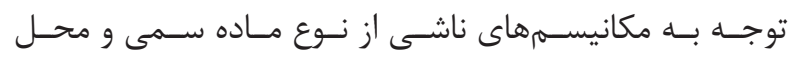

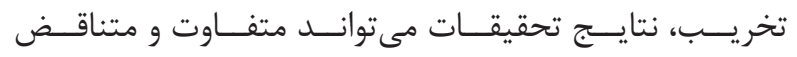

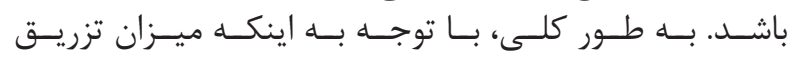

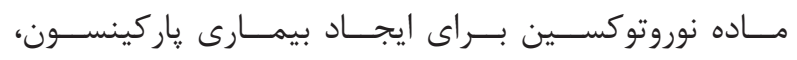

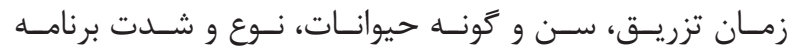

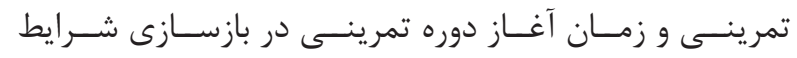

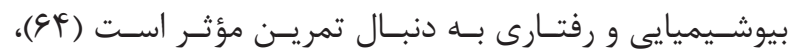

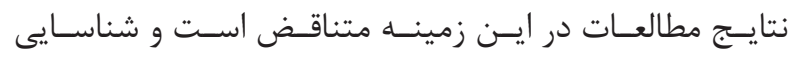

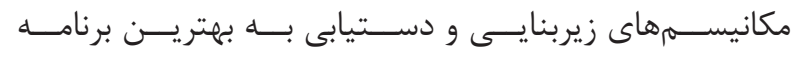

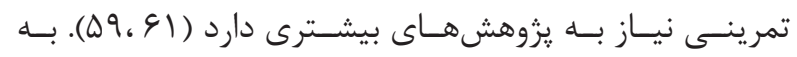

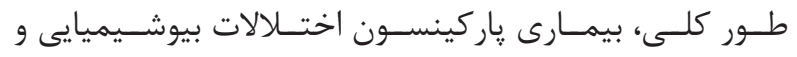

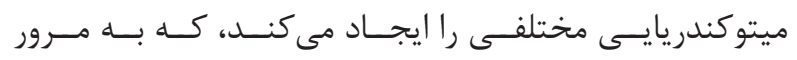

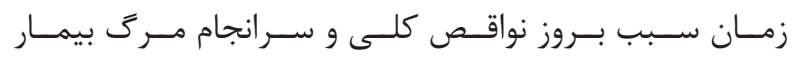

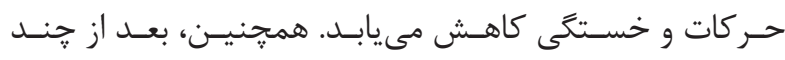

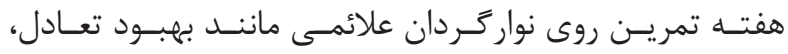

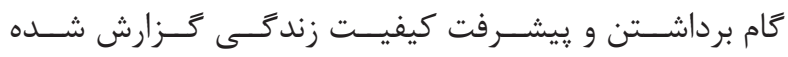

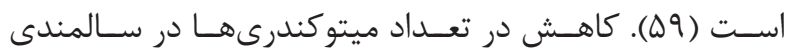

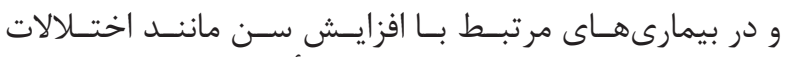

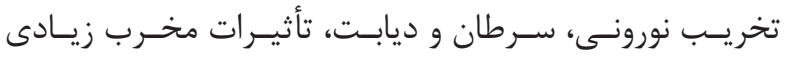

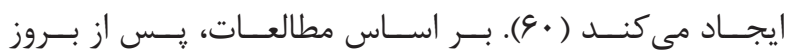

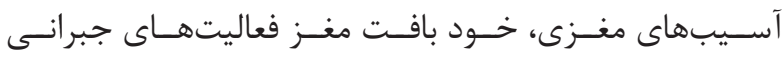

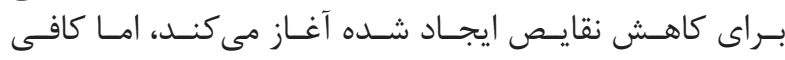

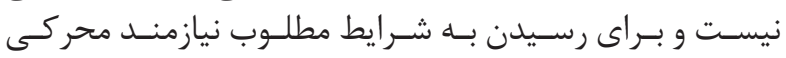

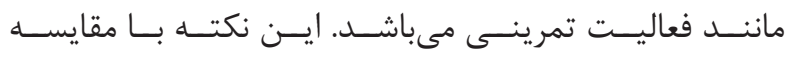

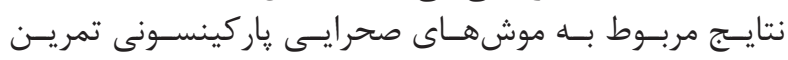

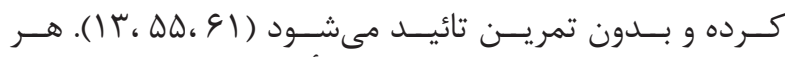

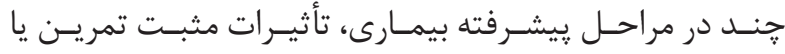

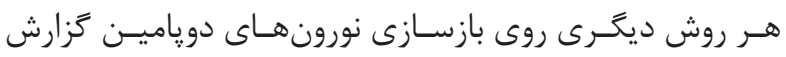

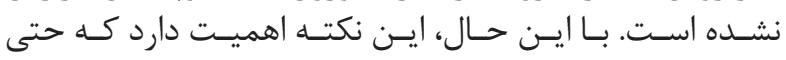

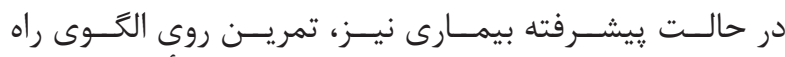

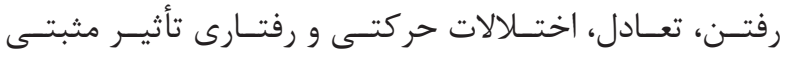

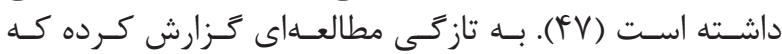

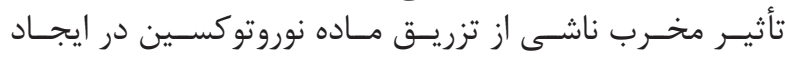

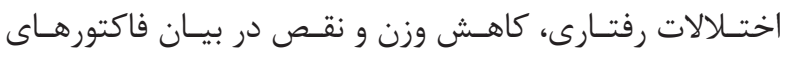

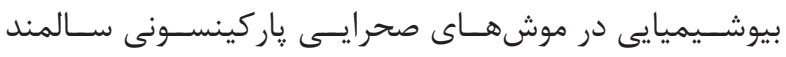

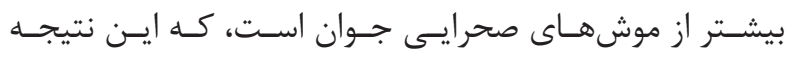

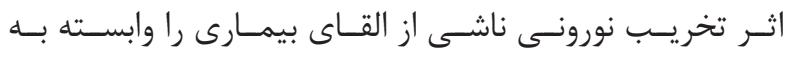

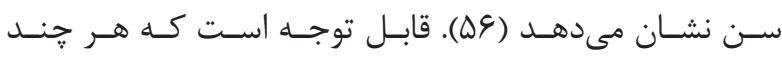

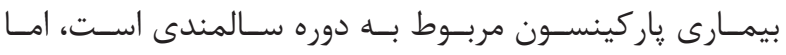

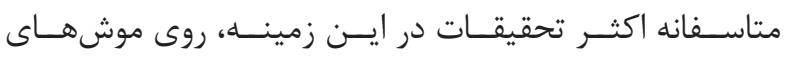

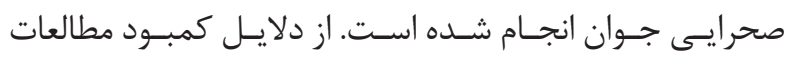

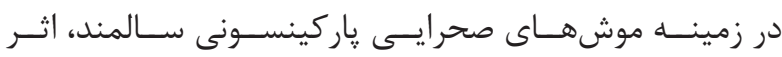

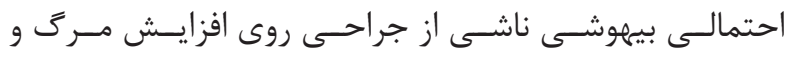

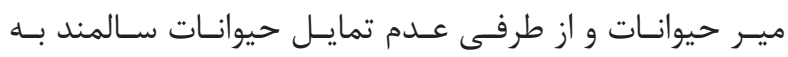

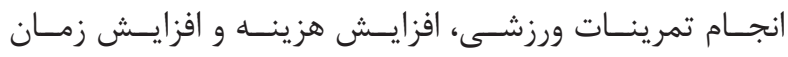

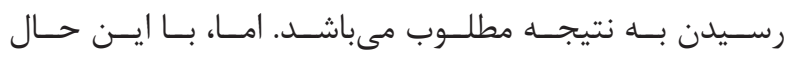

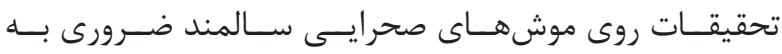

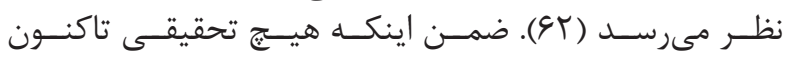

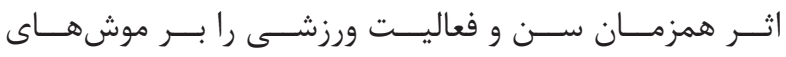

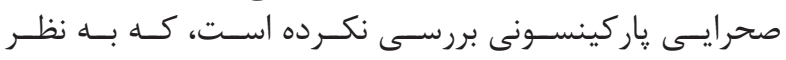

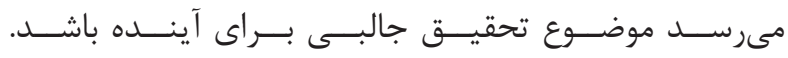

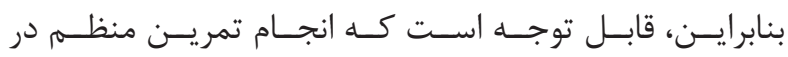

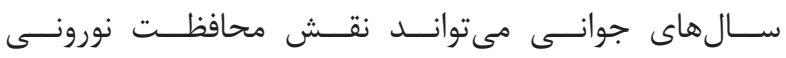

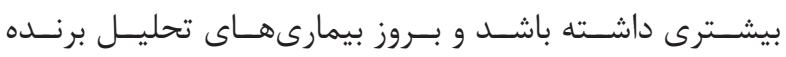

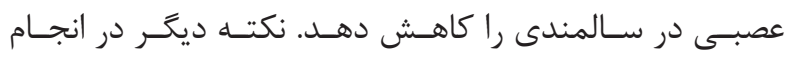

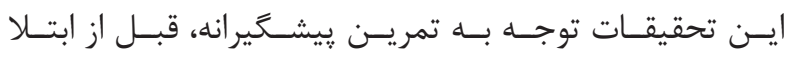

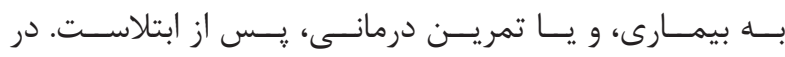

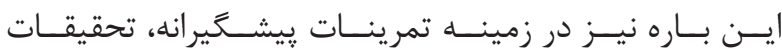

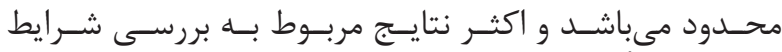

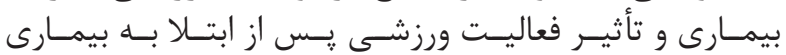

${ }^{38}$ Garcia 


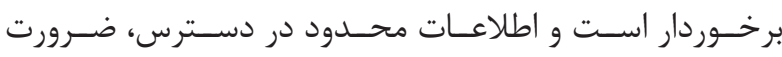

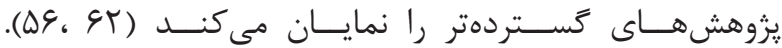

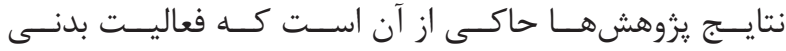

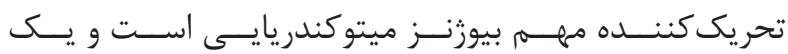

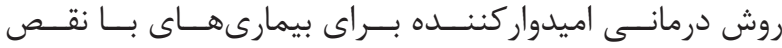

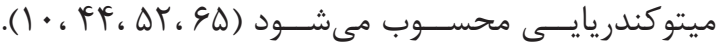

1. Smith BA, Goldberg NR, Meshul CK. Effects of treadmill exercise on behavioral recovery and neural changes in the substantia nigra and striatum of the 1-methyl-4-phenyl-1， 2， 3， 6-tetrahydropyridinelesioned mouse. Brain Res 2011; 1386: 70-80.

2. Lau Y-S, Patki G, Das-Panja K, Le W-D, Ahmad SO. Neuroprotective effects and mechanisms of exercise in a chronic mouse model of Parkinson's disease with moderate neurodegeneration. Eur J Neurosci 2011; 33(7): 1264-74.

3. Farshbaf MJ, Ghaedi K, Megraw TL, Curtiss J, Faradonbeh MS, Vaziri P, et al. Does PGC1 $\alpha /$ FNDC5/ BDNF elicit the beneficial effects of exercise on neurodegenerative disorders? Neuromol Med 2016; 18(1): 1-15.

4. Murray, D.K .The effects of exercise on cognition in Parkinson's disease: a systematic review. Transl. Neurodegener 2014. 3(1): 5-11.

5. Fujita KA, Ostaszewski M, Matsuoka Y, Ghosh $\mathrm{S}$, Glaab E, Trefois $\mathrm{C}$, et al. Integrating pathways of Parkinson's disease in a molecular interaction map. Mol neurobiol 2014; 49(1): 88-102.

6. LaHue SC, Comella CL, Tanner CM. The best medicine? The influence of physical activity and inactivity on Parkinson's disease. Mov Disorder 2016; 31(10): 1444-54.

7. Dauer W, Przedborski S. Parkinson's disease: mechanisms and models. Neuron 2003; 39(6): 889-909.

8. Subramaniam SR, Chesselet M-F. Mitochondrial dysfunction and oxidative stress in Parkinson's disease. Prog. Neurobiol 2013; 106: 17-32.

9. Autry AE, Monteggia LM. Brain-derived neurotrophic factor and neuropsychiatric disorders. Pharmacol Rev 2012; 64(2): 238-58.

10. Hou L, Chen W, Liu X, Qiao D, Zhou F-M. Exercise-

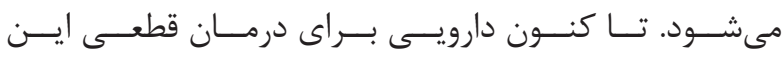

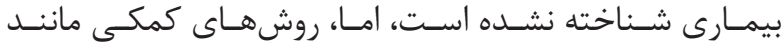

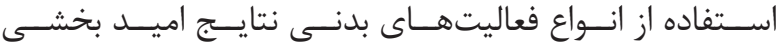

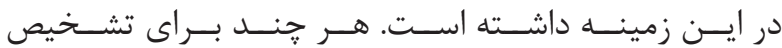

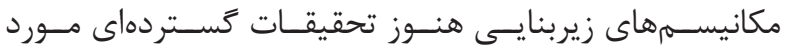

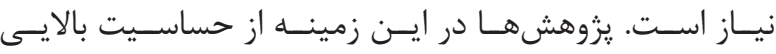

منابع

Induced Neuroprotection of the Nigrostriatal Dopamine System in Parkinson's Disease. Front Aging Neurosci 2017; 9(358).

11. Carta AR, Pisanu A, Carboni E. Do PPAR-Gamma Agonists Have a Future in Parkinson's Disease Therapy? Parkinson's Dis 2011; 2011: 689181.

12. Gerecke KM, Jiao Y, Pani A, Pagala V, Smeyne RJ. Exercise protects against MPTP-induced neurotoxicity in mice. Brain Res 2010; 1341: 72-83.

13. Rezaee Z, Marandi SM, Alaei H, Esfarjani F, Feizollahzadeh S. Effects of Preventive Treadmill Exercise on the Recovery of Metabolic and Mitochondrial Factors in the 6-Hydroxydopamine Rat Model of Parkinson's Disease. Neurotox Res 2019; 35(4): 908-17.

14. Rezaee Z, Marandi S-M, Ghaedi K, Esfarjani F. Molecular Mechanisms of Neurotrophins Actions on Diseases of Nervous System. Genetics in the Third Millennium 2015; 12(4): 3778-93 (in persian).

15. Aguiar AS, Castro AA, Moreira EL, Glaser V, Santos AR, Tasca CI, et al. Short bouts of mild-intensity physical exercise improve spatial learning and memory in aging rats: involvement of hippocampal plasticity via AKT, CREB and BDNF signaling. Mech Ageing Dev 2011; 132(11): 560-7.

16. Aguiar CCT, Almeida AB, Araújo PVP, Abreu RNDC, Chaves EMC, Vale OC, et al. Oxidative Stress and Epilepsy: Literature Review. Oxidative Medicine and Cellular Longevity 2012; 2012.

17. Oliveira NR, Marques SO, Luciano TF, Pauli JR, Moura LP, Caperuto E, et al. Treadmill Training Increases SIRT-1 and PGC- $1 \alpha$ Protein Levels and AMPK Phosphorylation in Quadriceps of Middle-Aged Rats in an Intensity-Dependent Manner. Mediators Inflamm $2014 ; 2014$.

18. Corona J, Duchen M. PPAR $\gamma$ and PGC- $1 \alpha$ as 
Therapeutic Targets in Parkinson's. Neurochem Res 2016: 1-9.

19. Kang C, Chung E, Diffee G, Ji LL. Exercise training attenuates aging-associated mitochondrial dysfunction in rat skeletal muscle: Role of PGC-1 $\alpha$. Exp Gerontol 2013; 48(11): 1343-50.

20. Richard M. Reznick HZ, Ji Li, Katsutaro Morino, Irene K. Moore, Hannah J. Yu, Zhen-Xiang Liu, Jianying Dong, Kirsty J. Mustard, Simon A. Hawley, Douglas Befroy, Marc Pypaert, D. Grahame Hardie, Lawrence H. Young, Gerald I. Shulman. Aging-Associated Reductions in AMP-Activated Protein Kinase Activity and Mitochondrial Biogenesis. Cell Metab 2007; 5(2): 151-6.

21. Wrann Christiane D, White James P, Salogiannnis J, Laznik-Bogoslavski D, Wu J, Ma D, et al. Exercise Induces Hippocampal BDNF through a PGC-1 $\alpha /$ FNDC5 Pathway. Cell Metab 2013: 18(5): 649-59.

22. Huh JY, Mantzoros CS. Irisin physiology, oxidative stress, and thyroid dysfunction: What next? Metabolism. 2015; 64(7): 765-7.

23. Jafari M, Farzanegi P. Changes in FNDC5 Expression in Heart Tissue of Old Rats Following a Course of Mandatory Exercise. Int. Res. J. 2015; 9(6): 793-94.

24. Cohen AD. Role of Exercise and GDNF in an Animal Model of Parkinson's Disease: Implications for Neuroprotection: University of Pittsburgh; 2006.

25. Rezaee Z, Marandi SM, Alaei H, Esfarjani F. The effect of preventive exercise on the neuroprotection in 6-hydroxydopamine-lesioned rat brain. Appl Physiol Nutr Metab 2019; 44(12): 1267-75.

26. Mooren F. Molecular and cellular exercise physiology. Human Kinetics; 2005.

27. Rezaee Z, Marandi SM, Alaei H, Esfarjani F. The effect of short endurance training on PGC-1 $\alpha /$ FNDC5/ BDNF signalling pathway in 6-OHDA-induced Parkinson's rats. Journal of applied exercise physiology 2019; 15(29): 15-26 (in Persian).

28. Sleiman SF, Henry J, Al-Haddad R, El Hayek L, Haidar EA, Stringer T, et al. Exercise promotes the expression of brain derived neurotrophic factor (BDNF) through the action of the ketone body $\beta$-hydroxybutyrate.
Elife 2016; 5: e15092.

29. Rezaee Z, Marandi SM, Ghaedi K, Esfarjani F. A review on the function and molecular structure of neurotrophins. Genetics in the Third Millennium 2015; 12(4): 3806-22.

30. Binder DK, Croll SD, Gall CM, Scharfman HE. BDNF and epilepsy: too much of a good thing? Trends Neurosci 2001; 24(1): 47-53.

31. McGough E, Kirk-Sanchez N. Exercise Interventions Targeting Neuroplasticity and Neuroprotection in Adults with Neurodegenerative Diseases. Neurodegener Dis 2012.

32. Salamon A, Torok R, Sumegi E, Boros F, Pesei ZG, Fort Molnar M, et al. The effect of physical stimuli on the expression level of key elements in mitochondrial biogenesis. Neurosci. Let. 2019; 698: 13-8.

33. Lima Giacobbo B, Doorduin J, Klein HC, Dierckx RAJO, Bromberg E, de Vries EFJ. Brain-Derived Neurotrophic Factor in Brain Disorders: Focus on Neuroinflammation. Mol. Neurobil. 2019; 56(5): 3295-312.

34. Snow W.M. Morris Water Maze Training in Mice Elevates Hippocampal Levels of Transcription Factors Nuclear Factor (Erythroid-derived 2)-like 2 and Nuclear Factor Kappa B p65. Frontiers in Molecular Neuroscience, 2015 8(70).

35. Yang X, Martin TA, Jiang WG. Biological influence of brain-derived neurotrophic factor (BDNF) on colon cancer cells. Exp Ther Med 2013; 6(6): 1475.

36. Landers MR, Kinney JW, Allen DN, van Breukelen F. A comparison of voluntary and forced exercise in protecting against behavioral asymmetry in a juvenile hemiparkinsonian rat model. Behav Brain Res 2013; 248(0): 121-8.

37. Bayod S, Del Valle J, Lalanza J, Sanchez-Roige S, de Luxan-Delgado B, Coto-Montes A, et al. Long-term physical exercise induces changes in sirtuin 1 pathway and oxidative parameters in adult rat tissues. Exp Gerontol 2012; 47(12): 925-35.

38. Psilander N. The effect of different exercise regimens on mitochondrial biogenesis and performance. 2014.

39. Dimatelis J, Hendricks S, Hsieh J, Vlok N, Bugarith 


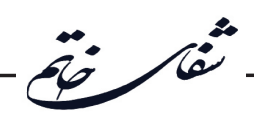

K, Daniels W, et al. Exercise partly reverses the effect of maternal separation on hippocampal proteins in 6OHDA-lesioned rat brain. Exp Physiol 2013; 98(1): 233-44.

40. Islam MR, Young MF, Wrann CD. The Role of FNDC5/Irisin in the Nervous System and as a Mediator for Beneficial Effects of Exercise on the Brain. Hormones, Metabolism and the Benefits of Exercise 2017: 93-102.

41. Yoon M-C, Shin M-S, Kim T-S, Kim B-K, Ko I-G, Sung Y-H, et al. Treadmill exercise suppresses nigrostriatal dopaminergic neuronal loss in 6-hydroxydopamine-induced Parkinson's rats. Neurosci Lett 2007; 423(1): 12-7.

42. Steiner JL, Murphy EA, McClellan JL, Carmichael MD, Davis JM. Exercise training increases mitochondrial biogenesis in the brain. J Appl Physiol 2011; 111(4): 1066-71.

43. Tuon T, Valvassori SS, Dal Pont GC, Paganini CS, Pozzi BG, Luciano TF, et al. Physical training prevents depressive symptoms and a decrease in brain-derived neurotrophic factor in Parkinson's disease. Brain Res Bull 2014; 108: 106-12.

44. Aguiar Jr AS, Duzzioni M, Remor AP, Tristão FSM, Matheus FC, Raisman-Vozari R, et al. Moderate-Intensity Physical Exercise Protects Against Experimental 6-Hydroxydopamine-Induced Hemiparkinsonism Through Nrf2-Antioxidant Response Element Pathway. Neurochem Res 2016; 41: 1-9.

45. Mabandla M, Kellaway L, Gibson ASC, Russell VA. Voluntary running provides neuroprotection in rats after 6-hydroxydopamine injection into the medial forebrain bundle. Metab Brain Dis 2004; 19(1-2): 43-50.

46. Tuon T, Souza PS, Santos MF, Pereira FT, Pedroso GS, Luciano TF, et al. Physical Training Regulates Mitochondrial Parameters and Neuroinflammatory Mechanisms in an Experimental Model of Parkinson's Disease. Oxid Med Cell Longev 2015; 2015.

47. Pothakos K, Kurz MJ, Lau Y-S. Restorative effect of endurance exercise on behavioral deficits in the chronic mouse model of Parkinson's disease with severe neurodegeneration. BMC Neurosci 2009; 10(1): 6.

48. Fu Y, Zhang Y, Yuan Q, editors. Aerobic exercise ameliorates learning and memory deficits of aging rats induced by D-galactose via promoting SYP and BNDF expression in hippocampus. BIO web conf 2017; 8 .

49. Deane KH JD, Ellis-Hill C, Clarke CE, Playford ED, Ben-Shlomo Y. A comparison of physiotherapy techniques for patients with Parkinson's disease. Cochrane Database Syst Rev 2001; 1.

50. Cho H-S, Shin M-S, Song W, Jun T-W, Lim B-V, Kim Y-P, et al. Treadmill exercise alleviates short-term memory impairment in 6-hydroxydopamine-induced Parkinson's rats. J Exerc Rehabil 2013; 9(3): 354.

51. Winklhofer KF, Haass C. Mitochondrial dysfunction in Parkinson's disease. BBA-MOL BASIS DIS 2010; 1802(1): 29-44.

52. Garcia PC, Real CC, Britto LR. The Impact of Short and Long-Term Exercise on the Expression of Arc and AMPARs During Evolution of the 6-Hydroxy-Dopamine Animal Model of Parkinson's Disease. J. Mol Neurosci 2017; 61(4): 542-52.

53. Poulton NP, Muir GD. Treadmill training ameliorates dopamine loss but not behavioral deficits in hemiParkinsonian rats. Experimental neurology. 2005; 193(1): 181-97.

54. Real CC, Garcia PC, Britto LRG. Treadmill Exercise Prevents Increase of Neuroinflammation Markers Involved in the Dopaminergic Damage of the 6-OHDA Parkinson's Disease Model. J Mol Neurosci 2017; 63(1):36-49.

55. Patki G, Lau Y-S. Impact of exercise on mitochondrial transcription factor expression and damage in the striatum of a chronic mouse model of Parkinson's disease. Neurosci let 2011; 505(3): 268-72.

56. Barata-Antunes S, Teixeira FG, Mendes-Pinheiro B, Domingues AV, Vilaça-Faria H, Marote A, et al. Impact of Aging on the 6-OHDA-Induced Rat Model of Parkinson's Disease. Int. J. Mol. Sci 2020; 21(10): 3459.

57. Soya H, Nakamura T, Deocaris CC, Kimpara A, Iimura M, Fujikawa T, et al. BDNF induction with mild exercise in the rat hippocampus. Biochem Biophys Res Commun 2007; 358(4): 961-7.

58. Miranda M, Morici JF, Zanoni MB, Bekinschtein P. Brain-Derived Neurotrophic Factor: A Key Molecule 
for Memory in the Healthy and the Pathological Brain. Front Cell Neurosci 2019; 13(363).

59. Salgado S, Williams N, Kotian R, Salgado M. An Evidence-Based Exercise Regimen for Patients with Mild to Moderate Parkinson's Disease. Brain Sci 2013; 3(1): $87-100$.

60. Rezaee Z, Marandi SM, Alaei H, Esfarjani F. The effect of endurance training on gene expression of PGC$1 \alpha$, BDNF and learning disorders in the hippocampus of aging rats. Sport physiology. 2019; 11(43): 91-104 (in Persian).

61. Fredriksson A, Stigsdotter IM, Hurtig A, EwaldsKvist B, Archer T. Running wheel activity restores MPTP-induced functional deficits. J Neural Transm 2011; 118(3): 407-20.

62. Pang SY-Y, Ho PW-L, Liu H-F, Leung C-T, Li L,
Chang EES, et al. The interplay of aging, genetics and environmental factors in the pathogenesis of Parkinson's disease. Transl. neurodegen 2019; 8(11): 23.

63. Carvalho MM, Campos FL, Coimbra B, Pêgo JM, Rodrigues C, Lima R, et al. Behavioral characterization of the 6-hydroxidopamine model of Parkinson's disease and pharmacological rescuing of non-motor deficits. Mol. neurodegener 2013; 8(1): 14.

64. Castren E, Pitkanen M, Sirvio J, Parsadanian A, Lindholm D, Thoenen $\mathrm{H}$, et al. The induction of LTP increases BDNF and NGF mRNA but decreases NT-3 mRNA in the dentate gyrus. Neuroreport. 1993; 4(7): 895-8.

65. Chen MJ, Russo-Neustadt AA. Exercise activates the phosphatidylinositol 3-kinase pathway. Mol Brain Res 2005; 135(1): 181-93. 